Témoigner Témoigner. Entre histoire et mémoire

Getuigen Revue pluridisciplinaire de la Fondation Auschwitz

$118 \mid 2014$

Au nom des victimes. Dictature et terreur d'État en Argentine, Chili et Uruguay

\title{
Mots du témoignage et de la mémoire
}

Words of testimony and of memory

Woorden van getuigenis en van herinnering

\section{(2) OpenEdition}

\section{Journals}

\section{Édition électronique}

URL : http://journals.openedition.org/temoigner/1282

DOI : $10.4000 /$ temoigner. 1282

ISSN : 2506-6390

Éditeur :

Éditions du Centre d'études et de documentation Mémoire d'Auschwitz, Éditions Kimé

Édition imprimée

Date de publication : 1 octobre 2014

Pagination : 200

ISBN : 978-2-84174-674-3

ISSN : 2031-4183

Référence électronique

"Mots du témoignage et de la mémoire », Témoigner. Entre histoire et mémoire [En ligne], 118| 2014, mis en ligne le 01 octobre 2015, consulté le 23 octobre 2020. URL : http://journals.openedition.org/ temoigner/1282 ; DOI : https://doi.org/10.4000/temoigner.1282 


\section{Dictionnaire testimonial et mémoriel}

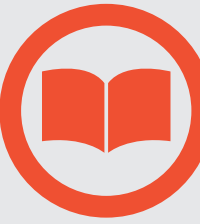

MOTS DU TÉMOIGNAG ET DE LA MEMOIRE

$\rightarrow$ Parce que les chercheurs, les enseignants et les profession des arts, de la culture et de

plus amenés à utiser des en plus artenans au champ du témoignage et de la mémor Témoigner entre histoire et mémoire s'est donné pour missio de les rassembler sous la forme un dictionnaire en ouvrant ainsi cet espace expérimenta.

$\diamond$ La réalisation de ce projet fait en deux temps. Chaque erme d'un index in progress la forme de notices courtes. d'abord dans chaque numéro de la revue, invitant ensuite a des développements et à une mise en débats critique, à plusieurs voix, sur un site qui 2014. À leur arsion colautom donc, volontairement partiell, nous associons quelques titres d'ouvrages ne prétendant pas à lexhaustivité.

\section{CASSANDRE}

assandre, fille du roi Priam apparaît brièvement dan des murailles de Troie, elle apostrophe ses compatriotes pour les op s̀ conpatiotes pour les retour du cadavre d'Hector. potentiel tragi ue lyrique se poit dévelop é utéringe se dans les tragédies. Prophétense dans les tragedies. Prophétesse l'Agrememnon d'Es (a partir de lint bon usage de sa raison (dai(a) elle devient une figure du savoir elle devient une figure du savoir inaudible puisque, sansjamais être sa cité et les horreurs de la guere. Esclave déportée à Mycèner dernier témoin du désastre, elle in incarne la chute de Troie par son solitude et sa fin tragique (elle mise ̀̀ et sa flyteme (elle est sureà mort par Clytennestre). En visionnaire lui permet même de témoigner d'un pé qué têmoigner dun passé qui ne lui tion is d'Eschyle, elle rappelle tion issue deschyle, elle rappelle

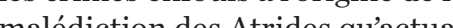
lisestion des d'Agmenouvelle fois lassassinat degamemnon par Clytemnestre Soulignant conis pávision

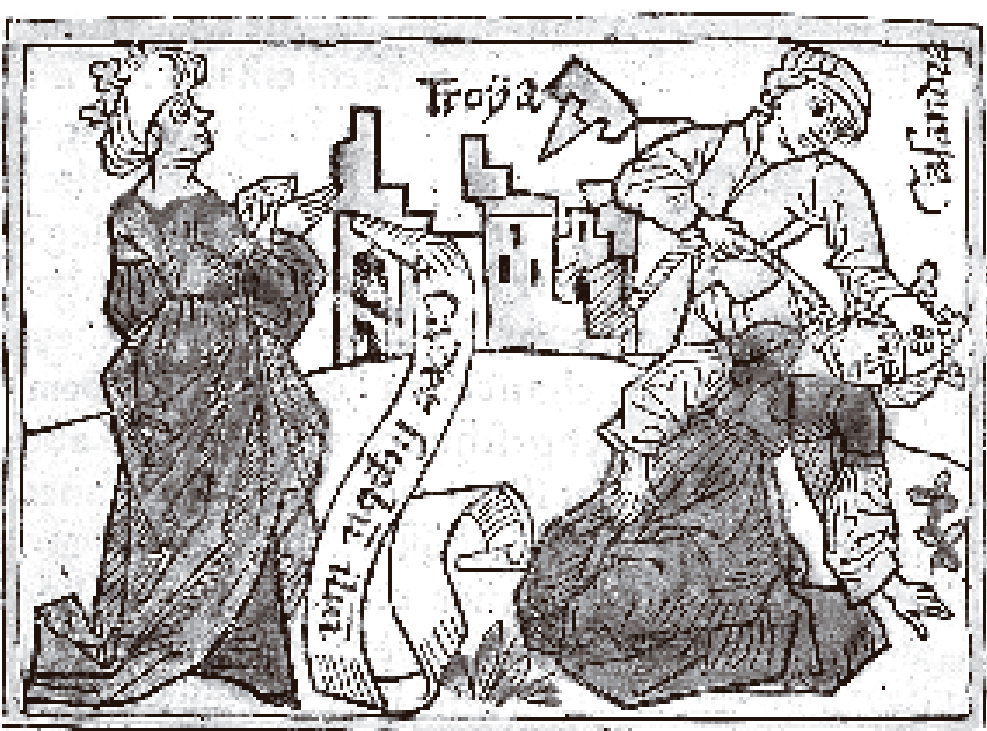

profondément mémoriel qu'est (Marthe, «mémoire inventive » parcel Detione nexistant que par sa réceptionet outildesymbotè corter témoi

Véronique Léonard-Roques
Blaise Pascal - CELIS EA 1002.

$$
\begin{array}{r}
\text { Université Blaise Pascal - CELIS EA 1002 } \\
\text { Clermont-Ferran }
\end{array}
$$

$\Delta$ Marie Goudot (dir.), Cassandre,

$\Leftrightarrow$ Véronique Léonard-Roques, Philippe Mesnard (dir.), Cassandre,

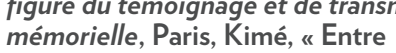
Histoire et Mémoire ", à paraît en 2015. $\diamond$ Romain Racine, "Cassandre », in
Pierre Brunel (dir.), Dictionnaire des mythes féminins, Monaco, Rocher,

René Schérer, Zeus hospitalier. Eloge de l'hospt. A Christa Wolf, Cassandre [édition par Alain Rance, Renate Lance
ÉCRIVAIN COMBATTANT

D

plupart des pays belgérants en 1914-1918, un

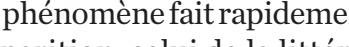
ture combattante Ses auteurs, selon les pays, sont appelés écriains - Frontdichter (Allemagne) ou Soldier-poets (Royaume-Uni) Ces dénominations désignent des Ces denominations désignent des du front, en ont tiré des œuvre littéraires et se sont constitués, à '’issue de la Première Guerre mondissue de la Preniere Guerremoncombattants sous l'égide de José Germain

e phénomène culturel se développa en raison de la mobilisation ou de l'engasentento taire massif des intellectuels dans taire massif des intellectuels dans se retrouvèrent ainsi sous l'uniforme Pares furent ceux qui chosirent de demeurer sile quichoiunérienter sure comme paroxysmique. Les livres qu'ils publiaient semblaient ains Túsés du sceau de l'authenticité. àne attente du grapd public à ane attente du grand public qui y trouvait une image de la guerre tages des journaux souvent assmilés au « bourrage de craneSoutenus par la critique, les éditeurs, les prix littéraires, les écriteurs, les prix litteraires, les écrHenri Barbusse furent vite rejoint par de plus jeunes auteurs, compattents jevenus écrivintains comme Maurice Genevoix Denevix ou Erist unger déconvirent

La poésie, le journal intime

La poesie, le journal intime et les avec des différences selon les avec des differences selon les paditions littéraires. La pés des cuerrefut ainsidavantagepŕsente on Allemane et au Royaume-Uni quelle ne le fut en From sielle n'é tait mas res le conflit, la reprise de l'expé lence de de deffet si le phénomènédito déclina apis 1918, il contort declina apres 1918, il connut un des années 1920 et 1930 avec la publication de romans de guerre remportan do ròns de guerre comme À l'Ouest rien de now

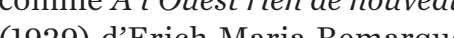
(1929) A 1,2 million dexences en allo 1,2 nillon dexenplaires cinques Les

Les textes des écrivains comliés a lew raterent il evis debats liés à leur statut, il y eut notamtion de la première étude critique du corpus français, Témoins (1929), par Jean Norton Cru, his (1929), pa Jeár Norton Cru, historien de 
combattant. Pour lui, la fonction estimoniale de ces textes primait le seule aune qu jugeait avec une grande sévérité les livres de guerre, pront peu de cas des intentions propres aux auteurs. Un certain Rorgès, répliqua verRoland Dorgeles, réphiqua vertenent, en mettant en avant dimensíńé par eux coureuvre, fondamentale que le témoignage. Nicolas Beaupré CHEC Clermont-Ferrand,IUF $\diamond$ Nicolas Beaupré, Écrits de la guerre "Biblis », 2013 [2006]

$\Delta$ Laurence Campa, Poètes de la combattante et activité poétique, Paris, Garnier, 2010.

$\diamond$ Pierre Schoentjes, Fictions de la

LÉCRITURE DU DÉSASTRE

ila notion de désastre évoque

la littérature apocalyptique comme genre et la tradition des Lane celles-ci (Jénie), en revanche, celles-ci n'entrent pas désastre dont la notion, en ta césastre dont la notion, en tant gue telle, revien à Maurce Blanment développée dans son recle ment développée dans son recuei L'Écriture du désastre (1980) Lériture du désastre (1980) paraissant sept ans apres Le Pas sorte de diptyque. Cette catégoó éme diptyque. Cette catégorie, èmergeant donc à ce moment intégrer les textes testim, vient integrer les textes testimoniaux e génocide.
Le désastre ne se réduit pas à (lysagelévénent commecatal’éche de Historire, mais àfaired désastre sle-même un lieu où le Plus qu'une éciture de survivant. Plus quine écriture de survivant du revent Langue antomatique

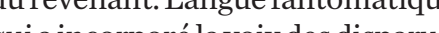

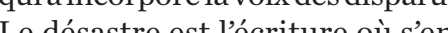

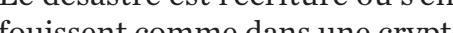
escendres de cux quirestents escendres decenxquirestentsans sepultre. Litteraturetestimoniale parce que testimoniale.

Le désastre est un mouvemen de pensée particulièrement complexe, car comme souvent che planchot, comme soút en para ésartroxes, voire en apories. Le représention du temps dans a repréntation du temps dans déjà eu lieu et, en même temps, equi est le plus proche tins, ,y a ni le plus proche. Ansi, n'y a ni espace, ni temps précis Mais le présent est le temps de la hais le présent est le temps de la i le temps lui-mêne peut faire où le temps lui-même peut faire le désastre Ainsi lapoclypse a to dous Ainsi, lapocalypse « Jojours dejan eu lieu bien que tou-

Lecriture du désastre met e scène une véritable poétique, qui priviegiéà Primo Levi dalanquelques mots d'introdu quelques mots dintroduction à $S$ cestun he lébris l'éclat iléch toutevo lébris, léclat, il échappe à toute volonté chronologique, a tout surable de la perte et son ressusement. dl déconte et son ressassenême déconstruit la possibilté êne de toute narration. À cet est particuliè de Chatotte elle exprime à travers soniferiture. poético-fragmentaire le bouleversement de la langue, traversée par l'horreur, confontée au muthe ou à son contraire, comm mutisme butiem à son disparition, à to Donner à toute disparition, à toute cendre, une gulière, telle pourrit tâche de l'é pour Ajoutons que philosophiqueAjoutons que philosophiquecrit dans la proximich de dans la proximite du concept le passivité tel que Levinas a pu lexposer dans Autrement qu'être ou au-delà de lessence, et ce, dan éch à toute forme d'expé échappe à toute forme d'expément, Lelós ment, selon le néologisme forgé pa
Blanchot.

$$
\begin{aligned}
& \text { Éric Hoppenot } \\
& \text { ESPE Paris IV La Sorbonne }
\end{aligned}
$$

$\diamond$ Paul Celan, Choix de poèmes, trad. Jean-Pierre Lefebvre, Gallimard,

$\triangle$ Charlotte Delbo, Aucun de nous

$\diamond$ Rachel Ertel, Dans la langue de personne? Poósie yiddish de

"Librairie du XX' siècle "1993.

$\diamond$ Sarah Kofman, Paroles suffoquées,

Pris, Galilée, 1987.

\section{THE GREY ZONE}

Un même mot peut prendre un sens différent
selon la langue dans laquelle il est convogué ou selon la langue dans laquelle lest convoque ou
il circule, cest pourquoi certains termes du dictionnaire seront abordés de façon
multilinguistique voire multiculturelle. C'est multilinguistique, voire
cas, ici, de la zone grise.

The 'grey zone' is a term coined by the Italian Holocaust survivor Primo Levi in his essay collection The Drowned and the Saved (1989; orlginally last book he completed before his death. In 'The Grey Zone', the second chapter and the longest essay in the book, Levi acknowledes the human need to divide the sociarly distin 'us' and then', we cleary distinct andidentinbinary thinking is inuthatsuch the face of the co indequate in in the camps ' [ [T] he in the camps. '[T] he network of human relationships inside the it could notbe reduced to the two it could notberednced to the two blocs of victims and persecutors (23). A key fact of Nazi practice, after all, was to attempt to turn victins into acc stoplices. Setting . separates (and not only in Nazi Lagers) the victins from the persectons (25), insight into which heconidens tobe of fundamental he by no Levi he by no distions in two categories: 'to con the two categories: 'to confuse [the moraldisese or an aesthetic affectationorasinistic tation or a snister sign of complicty; above all, it is a precious service rendered (intention' (33). The grey zone is inh

The grey zone is inhabited mostly by victims who comprooppressors to varying degrees and with varying varying degrees and f choice in exchange for prom of choice in exchange for preferone should refin fromists that asy judgment on these possing easy judgment on these morally who found theired prisoners ninferna thiron an infernal environment and who, olithic croup butcame in mandolithic group but came in many dierent shades of rey, with different he considers include low-rankin functionaries carrying out rou. and lice checking the Kapos of the work squads, the barracks chiefs, work squads, the barracks chiefs, diverse duties in who performed diverse duties in the Political Section, the Labour Service, Serve, and the punishment cells. the Sonderkommandos or 'special quis, entrusted with the running of the tate to call privileged. According to Levi, no pre is aud Aced the [ase individuals, who represent (34). Judgme (3).Jud, he argues, in the be susing cu, he Chaim, in the perplexcontroversial head of the Jewish councilin com exemplary occupant of the grey some length. While Levi primarily focuses on prile Leviphina oners in the camps and ghettos, his oners in thecamps and ghettos, his stretches to include collaborationthe Sontes paticular attention to

ist regimes such as those of Vichy France and Quisling in Norway an contempteds ming a youre chen alive from the gas chis It is a reflection, ultis che 作 and many differentcontexts, fields, and lisiplines, ranging from Hondisciplines, ranging from Hololow so to phing and culture.

Stef Craps
Ghent University

$\diamond$ Levi, Primo, 'The Grey Zone',

\section{inl., The Drowned and the Saved,}

Rosenthal, London: Abacus, 1986/1989, 22-51.

\section{LES JUSTES}

$\int \mathrm{d}$

aintenant plu e dix ans, les « Justes de Erance » sont régulièredel'État Aujourd'huivernaculaire le terme de Jasuivernacire, fait son entrée que récentant dans le vocabulaire national.

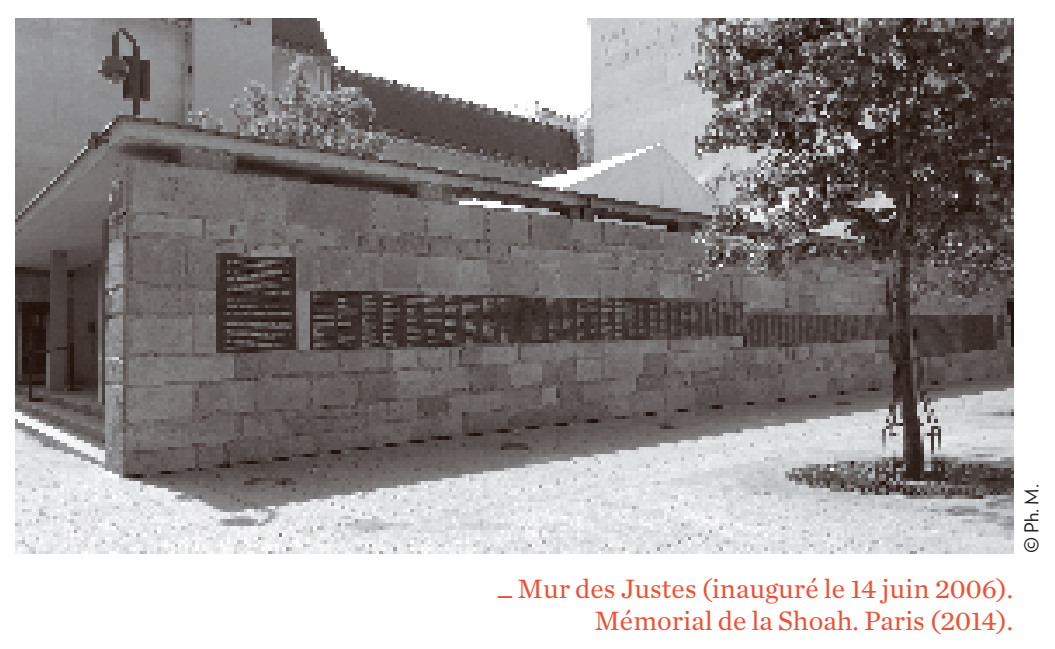


Le 19 août 1953, en Israël, les membres de la Knesset votent la création de l'Institut Yad Vashem fin de préserver le souvenir des martyrs et des héros du génocide reprenantune expression dorigine tén Nerion idédes Juifs au risquedeleurvie. Du fait des dificutés Du fait des difficultes mater rencontres par Yad Vashem, cette mission restepourtantlettre ctà la création d'un départem dédié délé aux Justes en 1963. Depuis lors, une com cours précidée rurlabe deux témoinagesde persones jux tur cos persontes juives qui considèrent lonnent lieu à la remise d'one

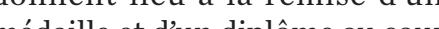
d'une cérémonie officielle assuré parle Ministère des Affaires étranparle Ministè des defire gères d'Israël.

Jusqu'au milieu des année 1980, peu de Français sont reconefois d'anciens résistants juifs se tefois, d'anciens resistants juifs se mobilisent pour faire connaitre ta la France compte 3760 Justes. Danslaprenicrepériode, lescérétaient rares et le plus souves étaient rares et le plus souvent organisées dans un lieu comdéroulent d́sormas presues tématiquement dans des me sytet bénéficient d'une las manies et bénéficient d'une large publicite. Én 1995, IAssenblee natioles sauveurs. historiquedul 6 juillet le précirst bén de la République, Jacques Chirac, reconnait les « Justes parmi les héros nationaux.
Ce discours marque le début la réappropriation progressive de la catégo hon progressive lenne l'État français. juillet 2000, le Parlement rebapJuse ainsi la loure du 16 jillet Journée comém crimes racistes et antisemites de l'́nes racistes et antisénites de "Justes" de Fra thommage aux "Justes" de France. » En janvier irn à lá Jacques Chirac inaug Shoah, plaue dans la cryptedu Panthé

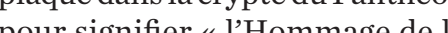
pation aux J l tes de Crandiose, la cér Trance lobjet d'un consensus politique. Élargissunt la netion isrá́lique. pour y inclure tous ceux qui son pour y inclure tous ceux qui sont encor, et resteront à jamais, ratif finit de faire entrer ceux qu nt désormais désignés du terne " "Juste" de France das de " Justes" de France » dan

Ces nouveaux héros y re

Ces nouveaux héros y remplissent la même fonction rhéPuisque fait historique avé trois-quarts des Jue avéré, les en la déportation, les quelquespé iers de Justes recolques Vashe Juses recriture Vashen ne constitueraient qu'une petite part de lensén qui ont é des sauvu sein de la popute majoritaires Cette seconde partie du raisone Cette s'a que peu de fondennehistoriographiques S'ilestcert. que les dossiers constitués certain che de la proćdure datribution du titre isrálien dán ient qu'une partie nes inerto qui ont effectivemes idé Juifs, les raisons de la survie des troisquarts des Juifs de France son encore peu nombreux et le plus souvent partiels, voire lacunaires de la socié frant la relate de la société française, I ampleu du territoire et l'importance de lespace rurá én son sein, loccupation differee de la zone sud ou encore lexistence de frontières avec des pays neutres, pour ne citer que quelques exemples, qui sont autant d' hypothèses aujourd hui considerees par les chercheurs. précède l'histoire. I

Sarah Gensburge
CNRS/ISP (Université Paris Ouest Nanterre) $\diamond$ Patrick Cabanel, Histoire des Justes $\diamond$ Sarah Gensburger, Les Justes de France. Politiques publiques de la 2010

$\diamond$ Jacques Sémelin, Persécutions Paris, Le Seuil, 2013.

\section{MÉMORIALISTE}

e mémorialiste porte témoignage de sa mémoire. On attend de lui qu'il fasse part l'une expérience exemplaire de phisité contemporaine, exemplarite que sa paroleconstruit dans la representation du rapport entre viduelle et le cénéral de l'hindviduelle et legencéral de lhistoire cui-même Que le mémorialiste ait lui-mêne trave façon remarquable comme témoin ou acteur dévenements narquants son écriture mais non nécessire: l'exemplarité de non parcours est travaillée par un récit qui protravaillée par un récit qui progroupe le mé de lindividu au montre en effet pas seul, mais lié

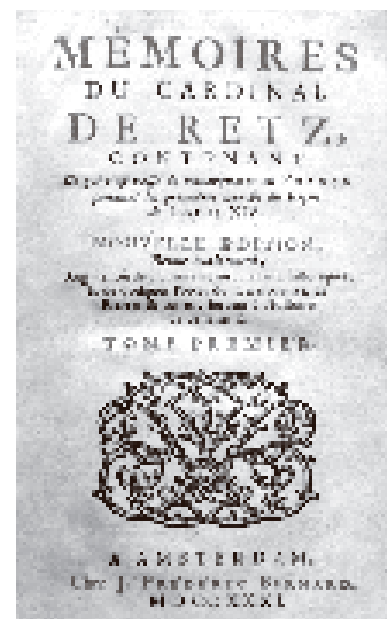

à ses contemporains ; il réfléchit aux aspects qui ont fixé les trait dominants de sa génération et a manière dont lui-même les uncarnés. Sa dénarche décriturese chue ainsi au croisement de récits collif été la solidarité entre les deux. Dans cette perspective, il peut ne pas sorhaiter satlarder sur les aspects pamiliales et sentimentales, for intérieur).

Depuis que les Mémoires sont identifiès comme une forme d'ectiture (en France, dans le courant d́x du rapport àlhistore ont pis tés du rapport a lhistoire ont pris bien des apparences, on peut citer otannentecardinal de Retzet e duc de Saint-Simon, les deux a période d'Ancien Régine: leprela période dAncien Régime:lepremier en est acteur et rend compte aux événements de la Fronpe, second en est témoin et se donne pour lon pour lobservateur minutieux des XIV. Après la Révolution Lous Roussea aussi, le témi mémorialiste s'affranchit progressivement: c'est aussibien en acteur qu'en témoin de l'histoire, en montrant la scène publique que le for ntérieur, pour écrire l'histoir Che pour illustrer la littérature que qui font de lui le morialiste de réć de luis la tration de reference dans la tradition historiqu. Depuis, le ténoignage

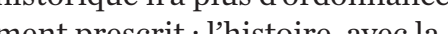
ment prescrit. Mhistoire, avec la grandehachen( de balayer les partages axcus en avait longtentares auxquels on avat longtenps cru en bouleve rend compte de ceux-ci comste d'u compte de ceux-ci conne dune expérence nourelle dont à réinven ex

Université comien Zanon Louvain-la-Neuve

$\diamond$ Jean-Louis Jeannelle, Écrire s au XXe siècle. Dédin "et renouveau, Paris, Gallimard,

$\triangle$ Jean-Louis Jeannelle, Marc Hersan \& Damien Zanone (dir.), Le Sens du
passé. Pour une nouvelle approche des Mémoires, in La Licorne. Revue de langue et de littérature françaises de
lUnniversité ede Poitiers, $\mathrm{n}^{\circ} 104$, Presses
universitaires de Rennes, 2013.

$\diamond$ Damien Zanone, Écrire son temps. Les Mémoires en France de 1815 à 1840 2006

\section{POSTMÉMOIRE}

e terme de postmémoir écrit la relation que la generation d'après » entrelectif et personn vécu par coqui l'ont précé í por cenx insi des expériences dont cette énération d'après ne se « souvient » que par le biais d'histoires, ves et de comportements parmi lesquels elle a grandi. Mais mises de faconsiplofonde tive qu' lles semblent propre mémoire. Le rappoit de la propre nénoire. Le rapport de vérité assuré par la médiation pas de souvenirs mais de projecpas de souvénis, hais de projecments imainats. Grundir ace lhéritage d’échar être dominé par des ścits qui ó précédé sa propee récts qui on propre conscien fart ou risque queleshisto fait courir vie soient elles-mênes déplás, voirént eles-mênes déplacées. C'estêtreformé bien qu'indirecte mentror ment, par des fragments traumatiques devenements qui défient etexcèdenta compréhension événements sont survenus dans passé, mais sours suffets contin le

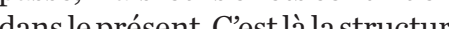
de laprént. Cestla lastructure proprè sa génération propre à sa génération.

Cependant, la postmémoir n'est pas une position identitaire nelle de transmission ancrée da de multiples formes de médiade multiples formes de médiases moments les plus intimes, ses mocinée dans un imaginaire eolectif façonné par des strurtures lectif façonné par des structure d'imagination et de projection parunfonds d'histoires et d'ima e par un fonds dhistorres et dimages et la mise à disposition plus vaste de souvenirs individuels et faste liaux Alors, si nous ads liaux. Alors, si nous adoptons les expériencestraunatiques dautrus 
quelles nous pourrions avoir vécu, $\mathrm{s}$ ous les inscrivons dans l'histoir denotreproprevie, pouvons-nous pour autant le faire sans les initer

Cette question concerne

Cette question concerne processus didentification, dimagination et de projection auss des familles de rescapés, que des des familles de rescapes, que des nénération ou du rés de leur génération ou du réseau reladu traumatismet triosí, l'urgence tu le beson curstré ét lurgence et le besoin custre de savoir quelque bho du tirnats paìs la ême. Pour tracer les froniè

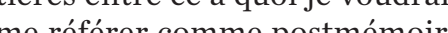
me riliére conne postménnoise lintion hation, hous devons tenir compte c ce qui distingue une ínentifeade varents à enfa l'intérieur de la cellus ant lieu àlias, d'une identificatio foniliale, dunécérationnelle faisontale tration de l'enfant est pue a position de lenfant est plus largement disponible a ce quilui vient de postmémoire por affilian. La postmémoire par affiliation céculte dénérans contenporaines et générationnelles avec la seconde genereration en tant que de structures de médiation de structures de médiation qui seraient amplement disponibles, appropriables et, en effet, plutôt "irrésistibles », pour englober un biologiqu de trans

Le « post » de postmémoi

Le «post » de postmèmoire
nnale plus qưun délai temporel et plus qu'une localisation dan un après-coup, ce n'est pas une à la temporalité linéaire, pas plus à la logique séquentielle. Considérons les nombreux «post » qui continuent de dominer champ intellectuel: postmoderexemple, intstructurasisme, par distance critique a interrelation respectivemis vec le modernisme et le structualis mon pas la fin du colonialisme, mais sa pas la fin du colonialsme, mais sa (c)

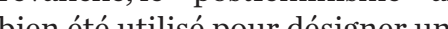
suite du féminis deus crainement encore dans lèr des des «post » qui - pour le meilproliférer : post-tramatique pien sûr, mais aussi « postläqun, bustain "posthumain ", postcolonial ", pestracial m. Rosalind Moris a céchment suggèré que le «post dhère à loomme un post-it qui

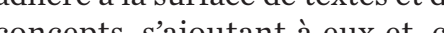
concepts, sajoutant à eux et, ce sorte de supprént den une Des postit bien sîr, souvent pro Dour destit, bien sur, souvent pris pour des pensées après-coup qui éconnecter de leur source. se déconnecter de leur source. lie doit persister portui-neept. eit, doit persister par lui-même pent cette position précaire il peut même acquérir ses propre

"Postmémoire » partage la stratification et le différé de ces autres « posts », s'alignant sur les plément qui les caroctéri du supmême que les autres “ posts même que les autres "posts" postnénoire» reflète une oscilet continuité De plus, « posturé et continuite. De plus, «postmémoire » n'st pas un mouvement, vis plutot come ou úc ; je le du retour inter- ou transgénérationnel d'un savoir traumatique et d'une expérience incorporée par ses destinataires. C'est une conséquence du rappel traumatique (different des troubles du stress post-traunatice), mais pris dans un mouvenent genérationnel Cemporel ou spatial.

Cette description de la structure de transfert inter- ou transgenérationnel du trauma souleve autant de questions qu'il apporte son lot de réponses. Pourquo pour décrire ces échanes? Si pour décrire ces échanges? Si la post l'esire dans lespace intime propre à la nismes, śtenent, par quels mécaplus distants, par adoption, ou a plus distants, par adoption, ou a Pourqui la postmér ajfinités? pourquì posticiénoire est-elle particulièrement liée au retour du reuse, ou bien concerner d'autre reuse, ou bien concerner d'autres types de changement historique qui, transmis à travers des générations, comportent la même intensite anbivalente? Quelles esthétiques, quelles technologies ou quels tropes peuvent-ils ou quels tropes peuvent-ils le la dimension pychologiqu pou postmémoire, les continuités et postmémoire, les continuités et les ruptures entre genérations, les lacunes du savoir, les peurs et les frayeurs qui découlent des conséquences du trauma? Et, pourquo phie en particulier, en shotograà jouer un rôle au, àjouer pajet

$$
\begin{array}{r}
\text { Marianne Hirsch } \\
\text { Columbia University, New York }
\end{array}
$$

(Traduit de l'américain par Philippe Mesnard)
REDIGNIFICATION

éologisme venu de l'espagnol (redignificacíon), la recessus par lequel la victime processus par lequel la victime
d'un crime violent pourrait dépasser cette condition et ce statut pour tenter de reprendre le contrôle de sa propre histoire. Utilisé et théorisé en Colombie par des anthropologues, des sociologues et psychologues travaillant avec les victimes des plus récents conflit armés colombiens (1980-2000), la redignification est l'un des objectifs principaux du travail de mémoire historique entrepris aux niveaux local et national, aussi bien par les associations de victimes, que par les instances et institutions publiques, notamment le Centre (Centro Nacío Centa: Nacionalde torica orica.gov.co).

Dans un contexte de violence généralisée, les violations des de la personne qui en de la personne qui en résulte d'humiliation et d'impuissone, dhuniliation et d'inpuissance les discours des actegitimés par mémoire estentendue ici, comme un processus permettant l'élaboration d'une identité à la fois indiviiturion stuation ruisent toriture moyen dit de redignification de victimes À partir d'exercices et soutien de partir detindidids

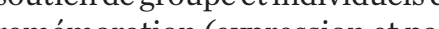
texpression et parentent de construire des discours center de constrire des discours libérer de ce qui les oppresse en appor de ce qui les oppresse en tation aux événements.

Le processus de redignificaLion est suppose se derouler en plusieurs étapes. La narration des souvenirs conduit à une prise de conscience de la condition de vicde lansi quá une connaissance lité des événe to Dès lou lite des éventer. Des lors, permetrat une rénterp pes expériene rénterprétation a latribution de significatice à lattribution de significations nouvelles, ce qui ferait naitre un Sentiment grâce auquel les vic Sentiment grâce auquel les vichontes de culles lesactes les discos criminels politiques les ont enfermées. Elles reprendraient alor vientrour , individes, ponvant revendique cursdroits à a rép revendique eraientcapables de mieuxdécide de lenteapables de mieux decider publique de la puit la de la ménoire entrainepolitique de la dounce sociale et politique da doulenrenturé, processus de redignification.

$$
\begin{array}{r}
\text { Marie Ines Harté } \\
\text { Facultad de Finanzas, Gobierno }
\end{array}
$$

y Relaciones Externado de Colombia

$\diamond$ Bello, Martha Nubia. (2001). "I Ientidad, dignidad y desplazamiento Desplazamiento forzado interno en Colombia : Conflicto, Paz y desarrollo,

$\rightarrow$ Grupo de Memoria Histórica CNRR (2009), Memorias en Tiempo Bogotá.

$\diamond$ Villa, IJuan David (2009), « La fuente de poder: un camino hacia la dignificación de las víctimas y la resistencia no violenta ", in ICTJ, 2009 Recordar en conflicto: iniciativas no

Bogotá. 
Site mémoriel

DOWTOWN MÉMORIEL
EN SAVOIR $\quad$ Georges Perec,
PLUS Robert Bober, Récits

d'Ellis Island, histoires
d'errance et d'espoir d'errance et d'espoir,
[1980], Paris, P.O.L., 1994. Pour le film
1980) Paris, P.O. 1994. Pour le film
- Michaël Prazan, Angel Island, l'autre

visage du rêve

américain, Arte - Les

Films du jour, 2014.
- Marita Sturken, Tourist of History Memory, Kitsch, and Consumerism from Oklahoma to Ground zero, Durham University Press, 2007

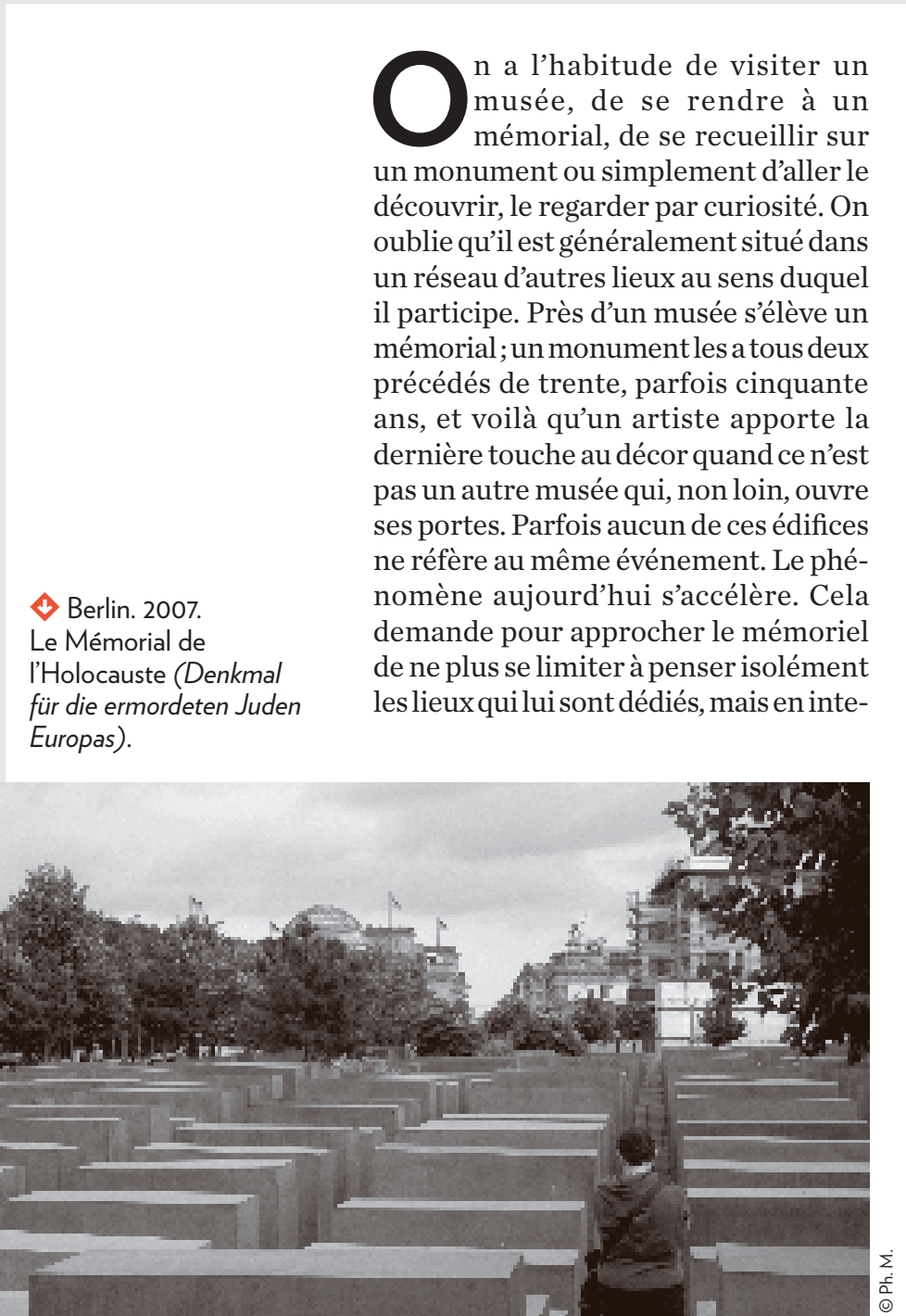

$\widehat{\text { Berlin. 2008. Façade gardant }}$ la trace des impacts d datant d'avril 1945

raction les uns avec les autres, de médir l'expérience même du parcours qui ène. Expérience de nos pas empruntés ers la mémoire, sur des chemins jalonés d'autres mémoires, d'intervalles, de furcations, d'oublis et de futilités, parflis qui peuvent etre bien nécessaires. Allez donc au Ménonial des Juif assassinés d Europe au cœur de Berlin. d'une desente en soi une entite chargée draze double histoire, celle du crime nazì celle des enjeux de sa mémoire après la rén ans de débats jusquen 2005 , linauguration). Mais on peut auss' s'interroge ur sa situation. L'on n'y accède pas sans avir dentrant complexe de structures, testrates et de passages porteurs dhistoire, de mémoire, de cultures, mais t' ville à ville qui garde encore aujourd hui su . du Mém davill 1945. L'enplacement du Mémorial aux Juifs assassinés deucope se trouve à la croisée de plusieurs chente bourg datant du début du XVIII avec son style néoclassique, un peu plus loin,

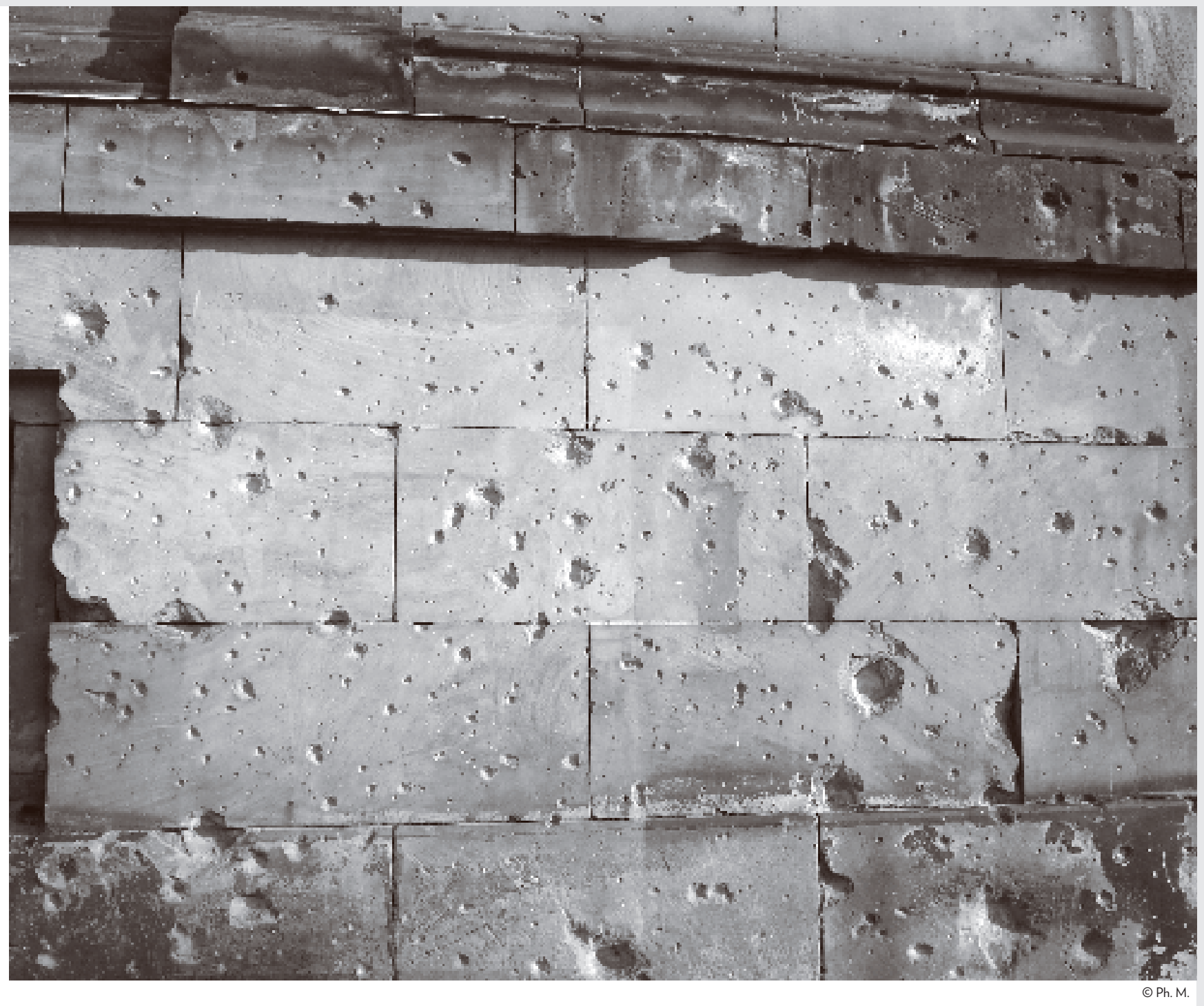




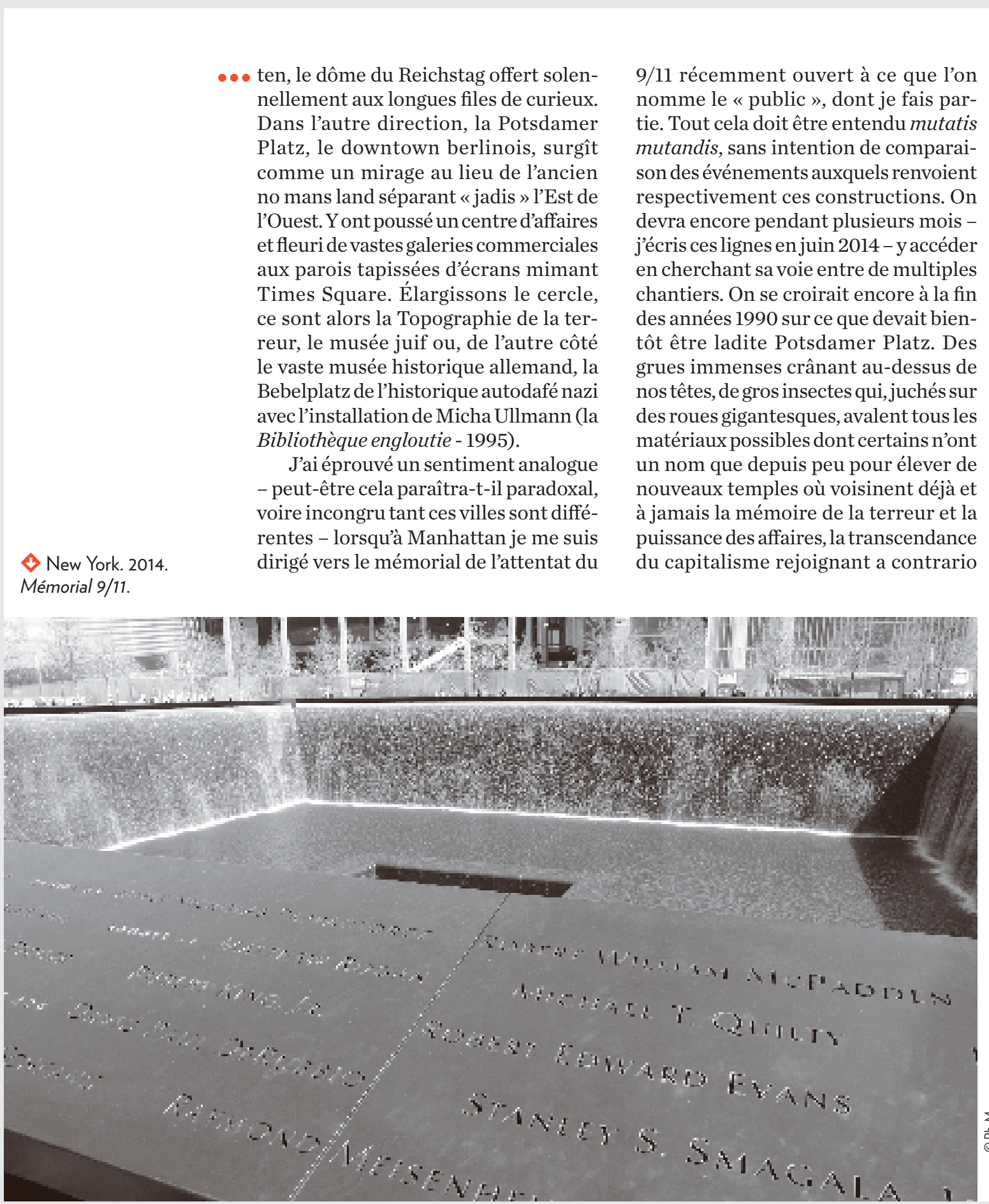

l'impossibilité - naturellement transcendante - de donner une mesure au tort subi

Nous passons dans un tourniquet puis empruntons une voie sinueuse soigneusement surveillée par des vigiles, des policiers et des gardes veillant autant à ce que personne ne s'égare qừ ce que l’ordre de notre écoulement, seuil après seuil, s'accomplisse consciencieucis où pour ế pour être vérifiè à nouveau jusquâtrois àl'int'par d'un scanner mine des pieds à late ne dispensant auces dis à latere ne dispensant der-avecla simplicitédectede améric la qui déconcerte politesse vient de France - si ce que, quand on dans la po in - que vous portez tons bis l'autre côté, ce sont bien des stylos. rautre cố, ce sont bien des stylos. Ne réellement le personnel de quassure il le joue aussi parfaitement et l'inte jou à mêne a la drate tègre par là même à la dramaturgie du sejo du public, res guides qui, à dispositio toiredel'arbrequi survécu ine incandescentes, ils conselllent et renjeu du même ordre on hez lesnatra un jeu du men ordre chez les guides en dans le musce d'Ellis Ishand (cettête, dans le musée d'elis Island (cette île, aujourd hui patringialisé, servit suivantdelieu d'examen pourator suivant de lieu dexamen pour autoriser cain des candidats à l'immigration cain des candidats à limmigration les plus nécessiteux).

L'esplanade du mémorial s'ouvr maintenant. Paradoxalement, l'effet est moins impressionnant que ce à quoi $j$

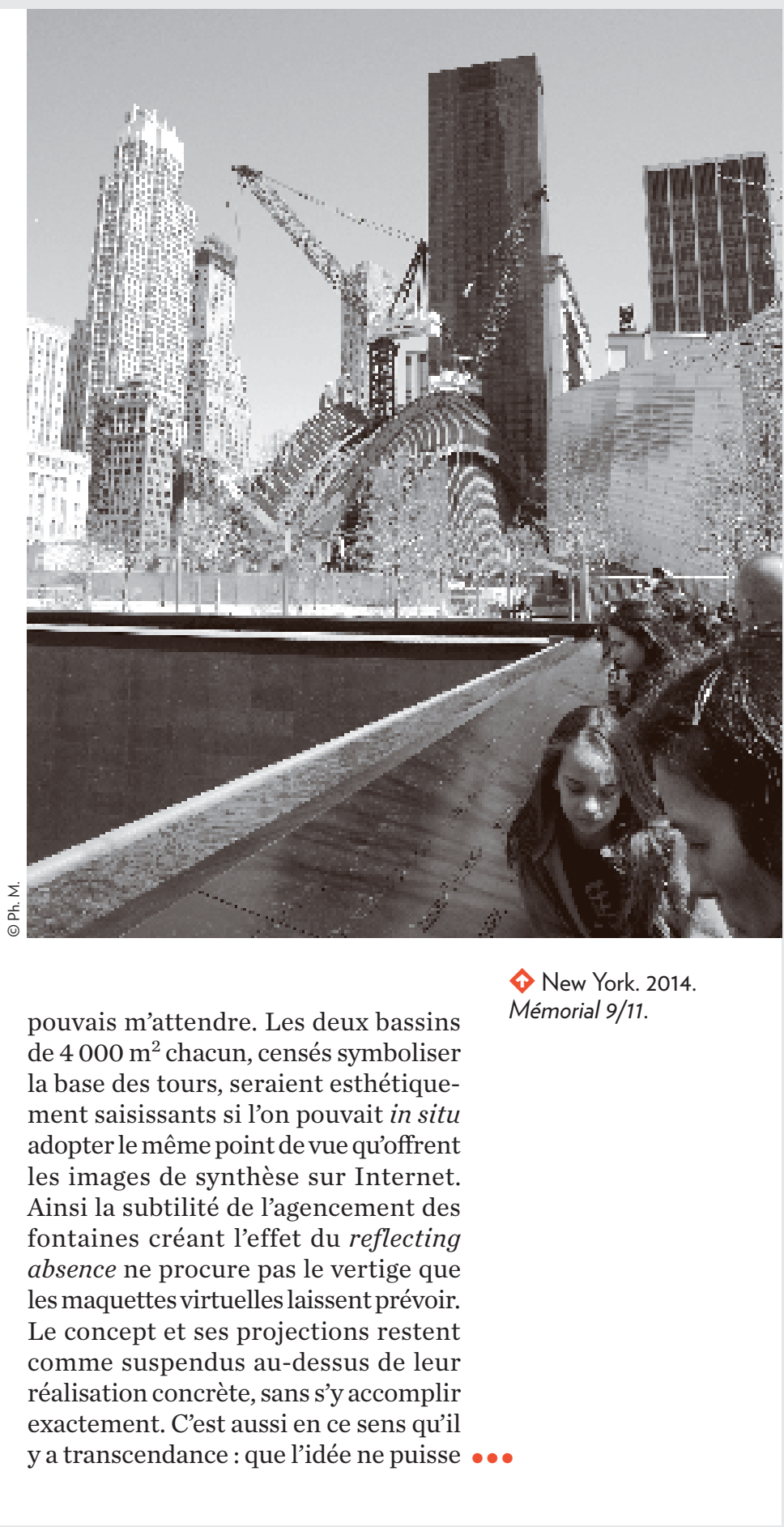




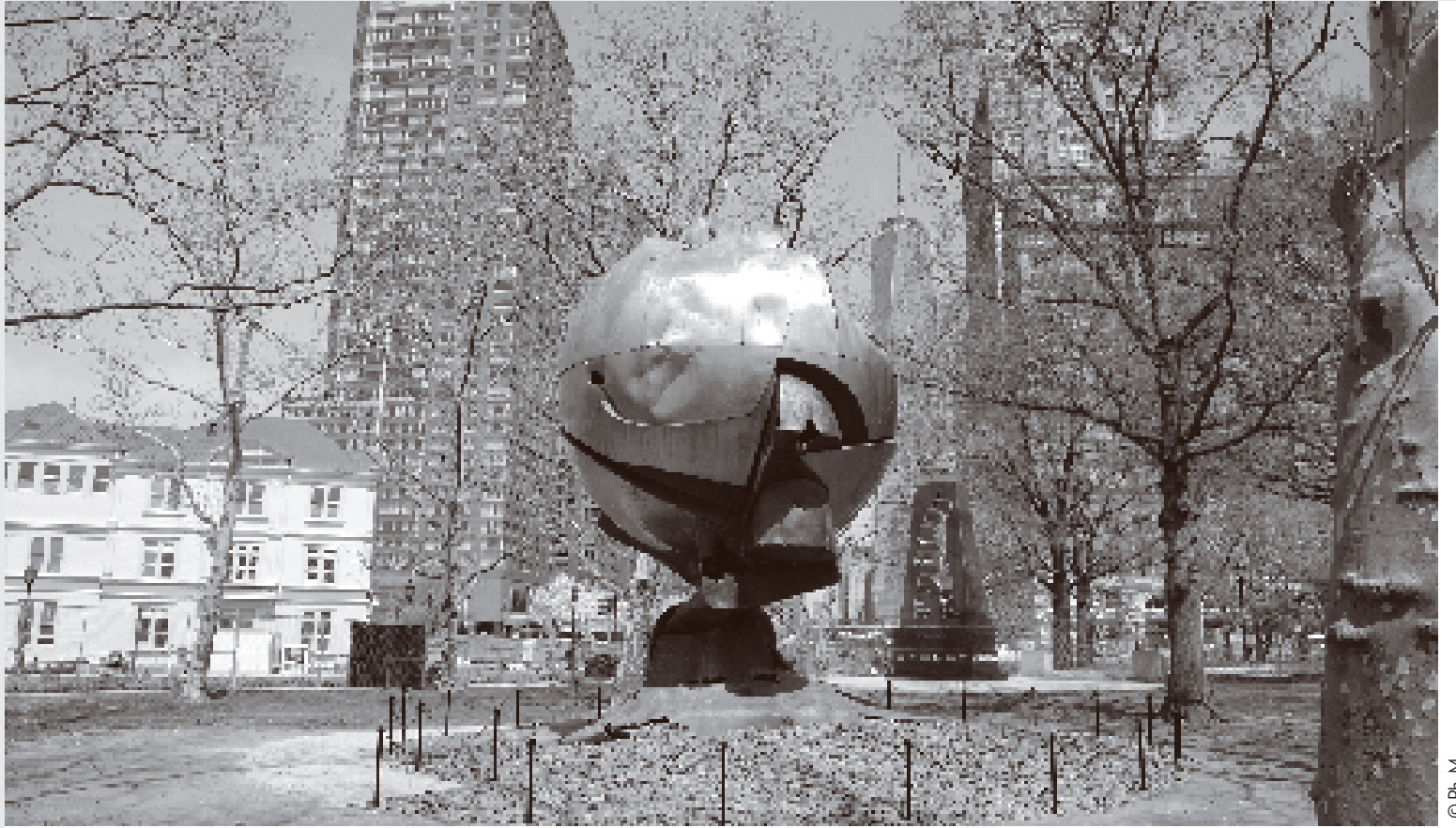

New York.

Battery Park. 2014.

de Fritz Koenig (1971,

à l'origine nommée

Grofe Kugelkaryatide)
déplacée après l'attentat sur les Twin. Juste derrière sur droite : le Corée de Mac Adams Corée de Mac Adams War Memorial). Dans le prolongement, au fond, la One World Trade Center.

coïncider avec sa réalité ; décalage qui répond certainement à la nécessité de

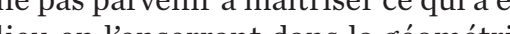
collective dusoun

De même qu’à Berlin le parcours entes stes du monument de i de dont aucher de devenir égales inter do devenir spectateur et, de nulle part, dispositif émaser, densemble du dispositif mémoriel, de même, les huit res 3 ond victines gravés sur les he rebor l'on bassins ne nous écrasent pas et lon ne peut totalité du site, bien que l'onent totalite du site, bien que lon ait le sentiment quil demeure à notre portée. Si grandeur ily a, cest que le nonum est en permanence élevé et porté par ménage urbain, se dégage un calme qui vous entraîne à lire les noms des morts, vous approcher de l'arbre survivant a sentir l'ar se cre lar sure nouvelle One World Trade Center de 510 mèt de haut dépassenter de ratte-ciel de New York et fint tous gratte-ciel de New York et, finalement, a percevoir le recueillement de la foule vrain quasinent palpable. Encela, le quis'y que l'intelligence même d'u bien plus que rint régé à mi dun dispositi hautement róc. Á mons que Tintelli-

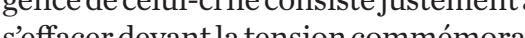
tive que cranta ive que propagent les présences venue Sortons

Sortons du mémorial du 9/11 pour rejoindre la définition plus étendue du site mémoriel que javais esquissée dessin. A la pointe de Downtown, se dessinerait un triangle venant en partie du Lower Manhattan. « Ground Zero en est la pointe intérieure, on l'aura deviné. Avant d'embarquer pour Ellis lsland, vous devrez prendre un ticIsland, les deux autres some et Ells tringle à l'envers qui donnets de triangle a l'envers qui done lon une Rien éride de la ter piérénateurs, ne justifie quat'o operateurs, nustis que ton alle seesoit curioux de la Statue (ce que lon soit curieux de la Statue (c'est un pe comme 1 " «ncontournable " triangle

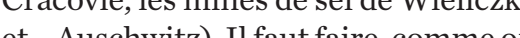
dit... de foch presios bien que fort do pression, bien que fort dépraisante aux oreilles, hestpent-ettrepassisotte. Dan la tête des agences, il ne faut plus

Ce viang de conber. Ce triangle mén êriel acquiert tout sonsens lo le cluide Battery Park dont pour Iibuty Island, cest avir pour Liberty Island, cest avoir préalabeins le gé pentant une heure au moins le ménorial aux soldats anercains morts durant la seconde Guerre tangulaires, quatre de cha stècéc un aigle victorie Sphere, le fameux globe de Fitz), The qui, ap te avor é durementa qui, après avoir été durentendon-

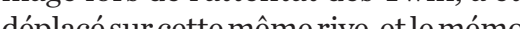
rià à gucte Cela fait Cela fait beaucous dan lement assez petit. Et si lon cherchait bans av sans avoir encore le pied sur le batean qui conduit à le delis, les nénoires quelques centaines de mitres sur lesquelques centahes de nètres sur lesquels se regroupenties files de touristes. sommet, Ellis Island.

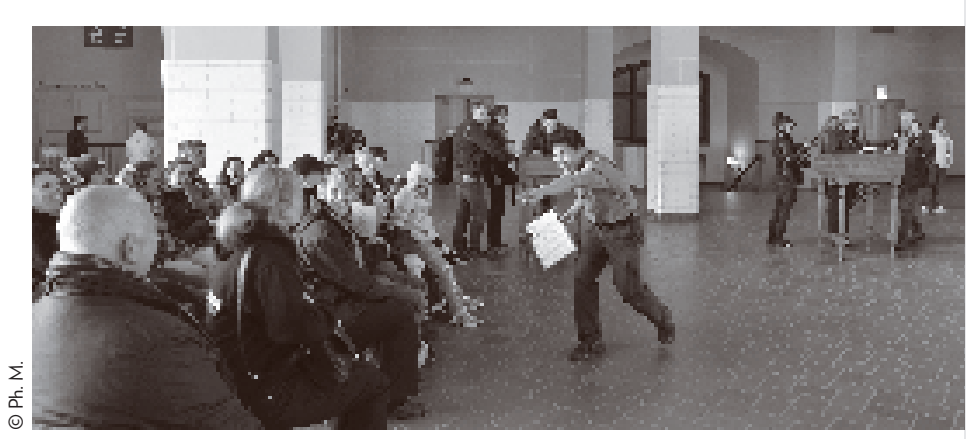

ث) Ellis Island. 2014.

Ellis Island est devenue un musée propre et très explicatif (ce qu'il n’était pastans emelin deś Ét láb́ct tantêt avec un guides jouent gratis, tique et soc un thousho dranalesque exaćé́, têle -j'en aifimé un lesque exageré, le roble -jen ai filmé un, sìcle de sentim de sentiment. Photos, objets, fac-similés ent quantité ne parviennent pourtant pas à nous rapprocher des murs de la vieille Europe atten les inmigrés nés, leur destin. Finterie lés, leur destin. Finalenent, la pièce la plus touchante ne donne pas grandchose à voir dautre que des moniteurs des affirent quasiment inut autour demeurent quasiment inaperçues) d'une même famille juatre personnes, plus, plus, tentent de retrouver un nom ou plusieurs sur des listes informatisées, oús. hés. Là encore, cesont des particuliers, appelons-les ainsi plutôt que visiteurs, qui donnent au dispositif général son atmosphère, une lueur que la scénoPhilippe Mesnard 